\title{
Chronic hyponatremia impairs memory in rats: effects of vasopressin antagonist tolvaptan
}

\author{
Toshiki Miyazaki ${ }^{1}$, Koji Ohmoto ${ }^{1}$, Tsuyoshi Hirose ${ }^{2}$ and Hiroyuki Fujiki ${ }^{1}$ \\ ${ }^{1}$ First Institute of New Drug Discovery and ${ }^{2} \mathrm{Qs}$ ' Research Institute, Otsuka Pharmaceutical Co., Ltd, 463-10 Kagasuno Kawauchi-cho, \\ Tokushima 771-0192, Japan \\ (Correspondence should be addressed to T Miyazaki; Email: t_miyazaki@research.otsuka.co.jp)
}

\begin{abstract}
The effects of stable chronic hyponatremia on the central nervous system are largely unknown, clinically, or in experimental animals. The aim of this study was to identify and characterize these effects in rats. Tolvaptan, a vasopressin $\mathrm{V}_{2}$ receptor antagonist, was used to correct hyponatremia and determine any potential benefits of such treatment in this condition. Stable chronic hyponatremia was induced by combination of the continuous vasopressin $\mathrm{V}_{2}$ receptor stimulation and liquid food intake. The hyponatremic rats did not exhibit significant changes in general symptoms or neurological functions assessed by modified Irwin's method, or in motor function assessed by the rotarod test. In passive
\end{abstract}

avoidance test, however, rats with moderate and severe hyponatremia had significantly reduced step-through latency, indicating impairment in memory. This reduced stepthrough latency was improved by the treatment of tolvaptan $\left(0 \cdot 25-8 \mathrm{mg} / \mathrm{kg}\right.$ daily doses), a vasopressin $\mathrm{V}_{2}$ receptor antagonist. This improvement is associated with normalization of plasma sodium concentrations in hyponatremic rats. In conclusion, these data suggest that chronic hyponatremia may impair memory, and treatments that normalize sodium level, such as vasopressin $\mathrm{V}_{2}$ receptor antagonists, may be beneficial to patients with hyponatremia.

Journal of Endocrinology (2010) 206, 105-111

\section{Introduction}

Hyponatremia is the most common disorder of body fluid and electrolyte balance and is associated with a variety of underlying disease states (Miller 2006). It has been reported that the symptoms of hyponatremia depend on the magnitude of low sodium level and the rapidity of its onset. Acute severe hyponatremia, which develops over $<48 \mathrm{~h}$, is often symptomatic and leads to neurological symptoms or death, in part because the resultant hypoosmolality produces brain edema (Soupart \& Decaux 1996). On the other hand, stable chronic hyponatremia that develops over periods of more than $48 \mathrm{~h}$ is believed to be asymptomatic in many cases due to brain adaptation to hypoosmolality (Verbalis \& Gullans 1991). Therefore, few studies have demonstrated the effects of chronic hyponatremia on the function of central nervous system (CNS) in either experimental animals or clinical studies. Several clinical studies have indicated that patients with mild to moderate chronic hyponatremia may experience attention deficits and gait disturbance, as associated with large bone fractures in the elderly (Renneboog et al. 2006, Sandhu et al. 2009). However, conclusive evidence is lacking to link chronic hyponatremia to neurological dysfunction.

Arginine vasopressin (AVP) plays a major role in body fluid regulation via $\mathrm{V}_{2}$ receptors in the renal collecting ducts, and altered regulation of AVP is a major factor in developing hyponatremia (Miller 2006). Thus, $\mathrm{V}_{2}$ receptor antagonism is effective to correct for hyponatremia (Schrier et al. 2006, Verbalis et al. 2008). Tolvaptan is a potent, highly selective, and orally effective nonpeptide $\mathrm{V}_{2}$ receptor antagonist. It promotes aquaresis: an increase in urine volume and decrease in urine osmolality, increase in electrolyte-free water clearance, and increase in serum sodium concentration (Yamamura et al. 1998, Hirano et al. 2000). Recently, FDA approved tolvaptan for the treatment of hypervolemic and euvolemic hyponatremia including patients with heart failure, cirrhosis, and syndrome of inappropriate antidiuretic hormone (SIADH; FDA Labeling Information 2009). In clinical studies, tolvaptan treatment demonstrated a significant increase in serum sodium after 30 days of therapy in patients with euvolemic or hypervolemic hyponatremia (SALT-1 and SALT-2). Furthermore, there was a significant improvement in self-assessed mental health status (vitality, social functioning, emotionally limited accomplishment, calmness, and sadness) assessed by the Mental Component Summary of the SF-12 General Health Survey (Schrier et al. 2006). While these results suggest chronic hyponatremia may be associated with reduced quality of life (mental condition), more detailed studies are needed to fully understand the impact this condition on CNS function.

The aims of this study were to clarify these questions: 1) does chronic hyponatremia cause CNS-related changes in general symptoms and behavior (Irwin's assessment test), 
motor activity (rotarod treadmill test), and memory (passive avoidance test, PAV)? 2) does correction of hyponatremia using tolvaptan have any benefit in these evaluations?

\section{Materials and Methods}

\section{Animals}

The care and handling of animals were in accordance with the 'Guidelines for Animal Care and Use in Otsuka Pharmaceutical Co., Ltd (Tokushima, Japan); October 1, 1994'.

Male Sprague-Dawley rats (6 weeks old) were purchased from Charles River Japan, Inc. (Yokohama, Japan), and housed under conditions of: humidity, $60 \pm 10 \%$; temperature, $23 \pm 2{ }^{\circ} \mathrm{C} ; 12 \mathrm{~h}$ light: $12 \mathrm{~h}$ darkness cycle $(0700$ to $1900 \mathrm{~h}$ for the light period and 1900 to $0700 \mathrm{~h}$ for the darkness period) for 2 weeks before the experiments. They had free access to food and tap water before the experiments.

\section{Drugs}

[Deamino-Cys ${ }^{1}$, D-Arg ${ }^{8}$ ]-Vasopressin (DDAVP) was purchased from Sigma-Aldrich Japan Co., Ltd.

Hydroxypropyl methylcellulose (HPMC) was purchased from Shin-Etsu Chemical Co., Ltd (Tokyo, Japan). Tolvaptan (( \pm )-7-chloro-5-hydroxy-1-[2-methyl-4-(2-methylbenzoylamino)benzoyl]-2,3,4,5-tetrahydro- $1 H$-benzazepine) was prepared at Otsuka Pharmaceutical Co., Ltd, and was suspended in 1\% HPMC solution.

\section{Study 1: Assessments of CNS-related changes caused by hyponatremia}

To determine the effects of hyponatremia on CNS-related function, three groups of rats were studied: normonatremia, moderate hyponatremia, and severe hyponatremia.

Hyponatremia was induced by a method described elsewhere (Miyazaki et al. 2005). In brief, rats (8 weeks old) were implanted with osmotic minipumps (ALZET model 2002, DURECT Corporation, Cupertino, CA, USA) containing DDAVP $(0.3$ and $1 \mathrm{ng} / \mathrm{h}$ to produce moderate and severe hyponatremia respectively) or saline in the back of each rat under ether anesthesia on day 0 . Animals received $40 \mathrm{ml} /$ day of modified liquid food containing 14\% dextrose. The procedure for modified liquid food preparation was based on the 'Preparation of Liquid Food' (Oriental Yeast Co., Ltd, Osaka, Japan), but water was exchanged with a 14\% dextrose solution. Blood (about $0.3 \mathrm{ml}$ ) was collected from the tail vein using a capillary tube on days $0,5,9$, and 13 for plasma sodium measurement.

Irwin's test General symptoms and neurological function were evaluated on day 5 using modified Irwin's assessment test (Irwin 1968). All evaluations were performed by two researchers who were blinded to the treatments. The animals were individually placed in transparent plastic cages $(27 \cdot 3 \times 49 \cdot 1 \times 27 \cdot 3$ (height) $\mathrm{cm})$ containing the wire-mesh grid for observation. After the animals were acclimatized for at least $1 \mathrm{~h}$, general symptoms and neurological functions were determined by 38 assessments (respiratory rate, stereotypy, grooming, vocalization, restlessness, Straub tail, tremor, twitch, convulsion, body posture, writhing, body tone, piloerection, urination, defecation, aggression, touch response, alertness, palpebral opening, ptosis, startle response, passivity, grip strength, catalepsy, spontaneous activity, abnormal gait, exophthalmos, skin color, lacrimation, salivation, pupil size, corneal reflex, pinna reflex, pain response, ipsilateral flexor reflex, limb tone, righting reflex, and hypothermia).

Rotarod treadmill test On day 10, motor function was evaluated in a rotarod treadmill apparatus (MK-660D, Muromachi Kikai Co., Ltd, Tokyo, Japan). The rotarod test was performed by a method described elsewhere (Yu et al. 2007) with some modification. Rats were placed on a rotating drum (6 r.p.m., trained until animals could remain on the apparatus at least $1 \mathrm{~min}$ at the training session, 6-60 r.p.m. accelerated for $3 \mathrm{~min}$ at the testing session, cut-off time was $180 \mathrm{~s}$ ), and the time to maintain balance on the rotarod was measured.

Passive avoidance test PAV was performed by a method described elsewhere (Darbra et al. 2004) with some modification using an automatic-controlled, step-through type passive avoidance chamber (Yamashita-Giken, Tokushima, Japan) on days 12 and 13. The chamber consisted of two compartments made of acryl which separated a movable guillotine door; lighted room $(15 \times 20 \times 20$ (height) $\mathrm{cm})$, and the dark room $(20 \times 25 \times 20$ (height) $\mathrm{cm}$, the stainless steel grid floor which was supplied with an electric foot shock). In the training session (day 12), each rat was placed in the light compartment. After about $10 \mathrm{~s}$, the guillotine door was automatically elevated, and when the rat

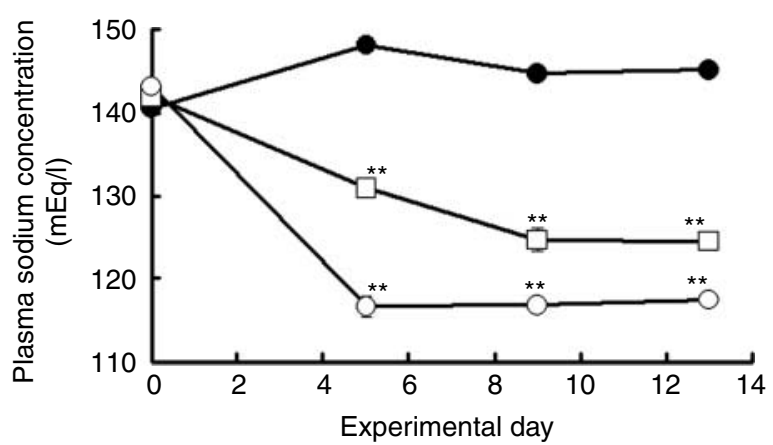

Figure 1 Plasma sodium levels during the experimental period. Values are expressed as the mean \pm s.E.M. of ten animals. ${ }^{* *} P<0 \cdot 01$ compared with normonatremia group by repeated measures ANOVA, followed by Dunnett's test at each time point. denotes normonatremia, $\square$ denotes moderate hyponatremia, $\bigcirc$ denotes severe hyponatremia. 


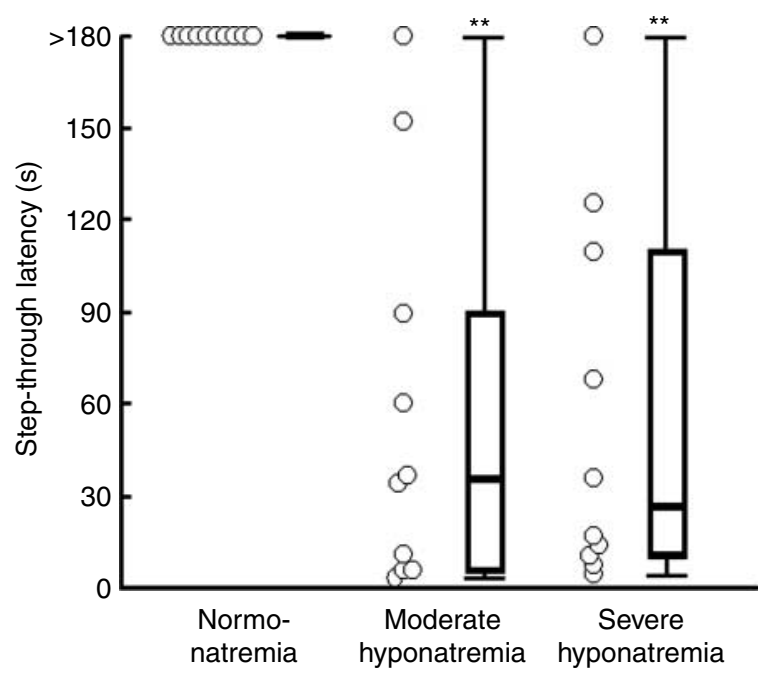

Figure 2 Effect of hyponatremia on step-through latency in passive avoidance test. The differences between the normonatremia and hyponatremia groups were analyzed by Steel's test. ${ }^{* *} P<0 \cdot 01$ compared with normonatremia group. The individual step-through latencies of ten animals (circle) and median with interquartile range (box and whisker) are shown.

moved into the dark compartment, the door was immediately closed, and 2-mA foot shock was applied through the grid floor for $3 \mathrm{~s}$. Twenty-four hours after the training session, the rat was returned to the light compartment and the time for the rat to move into the dark compartment was measured up to the maximum cut-off time (180 s). Step-through latency was measured as an index of memory.

Study 2: Effect of tolvaptan treatment on reduced step-through latency of hyponatremic rats in $P A V$

Three groups of rats were studied, normonatremia, hyponatremia (vehicle), and hyponatremia (tolvaptan). On day 0 , rats were implanted with osmotic minipumps containing DDAVP (1 ng/h, $n=20$ ) or saline (normonatremia, $n=10$ ). The rats in the hyponatremia group were further divided into the vehicle $(n=10)$ and tolvaptan $(n=10)$ groups by matching their individual body weight and plasma sodium concentrations measured on day 2 . Tolvaptan or the vehicle (1\% HPMC) was administered by dose titrations beginning on day 4. Administered doses of tolvaptan were as follows: $0 \cdot 25$ (day 4), $0 \cdot 5$ (day 5), $1 \cdot 0$ (day 6), $2 \cdot 0$ (day 7), $4 \cdot 0$ (day 8$)$, and $8 \cdot 0$ (days $9-13) \mathrm{mg} / \mathrm{kg}$. Vehicle was administered to the normonatremia group. Blood (about $0.3 \mathrm{ml}$ ) was collected from the tail vein using a capillary tube on days $0,2,4,7$, and 10 for plasma sodium measurement. PAV was performed on days 13 (training) and 14 (testing) using the method as described in Study 1. After PAV on day 14, blood samples were collected from the abdominal vein under anesthesia. All the animals were killed, and the brain was isolated for measuring brain water content. Isolated brain was weighed (wet weight), dried at $110^{\circ} \mathrm{C}$ for at least $48 \mathrm{~h}$ and weighed again (dry weight), and brain water content was calculated by the formula shown below:

Water content $(\%)=($ wet weight $(\mathrm{g})-$ dry weight $(\mathrm{g})) /$ wet weight $(\mathrm{g}) \times 100$.

Study 3: Effect of tolvaptan itself on step-through latency of normal rats in $P A V$

To determine whether tolvaptan itself influences memory, step-through latency of normal rats in PAV with/without tolvaptan was measured. PAV was performed as described above; however, tolvaptan $(10 \mathrm{mg} / \mathrm{kg})$ or $1 \%$ HPMC solution was orally administered $1 \mathrm{~h}$ before PAV, and $0 \cdot 8-\mathrm{mA}$ foot shock was applied for $2 \mathrm{~s}$.

\section{Statistical analysis}

Differences were considered to be statistically significant at $P<0 \cdot 05$.

Differences in plasma sodium concentration were statistically analyzed using repeated measures of ANOVA, followed by two-tailed Dunnett's test or two-tailed $t$-test. Differences in step-through latency were analyzed by two-tailed Steel's test, Wilcoxon's rank sum test, or Steel-Dwass's test. Differences in other parameters were analyzed by two-tailed $t$-test.

\section{Results}

Study 1: Assessments of CNS-related changes caused by hyponatremia

Stable and chronic hyponatremia were created by combination of the continuous vasopressin $V_{2}$ receptor stimulation and the excessive water loading using liquid food intake. By day 5 , mean plasma sodium concentrations

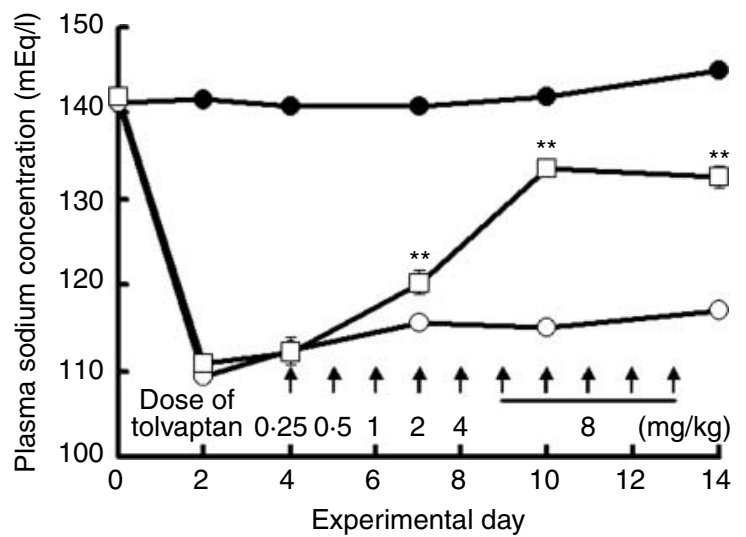

Figure 3 Effect of tolvaptan on plasma sodium levels in chronic hyponatremia. Values are expressed as the mean \pm S.E.M. of ten animals. ${ }^{* *} P<0 \cdot 01$ compared with hyponatremia vehicle group by repeated measures ANOVA, followed by two-tailed $t$-test at each time point. denotes normonatremia, $\bigcirc$ denotes hyponatremia vehicle, $\square$ denotes hyponatremia tolvaptan. 


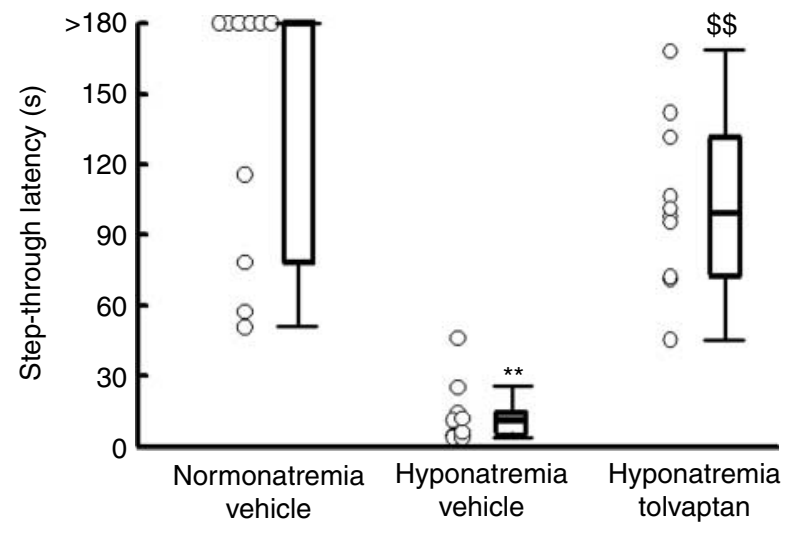

Figure 4 Effect of tolvaptan on step-through latency in passive avoidance test in hyponatremic rats. Step-through latency was measured up to the maximum cut-off time (180 s). The differences between the normonatremia and the hyponatremia vehicle, and between the hyponatremia vehicle and hyponatremia tolvaptan groups were analyzed by Wilcoxon's rank sum test. ${ }^{* *} P<0 \cdot 01$ compared with normonatremia group. ${ }^{\$} \$<<0 \cdot 01$ compared with hyponatremia vehicle group. The individual step-through latencies of ten animals (circle) and median with interquartile range (box and whisker) are shown.

decreased to $130.9 \pm 0.8 \mathrm{mEq} / 1$ in moderate hyponatremia (DDAVP: $0.3 \mathrm{ng} / \mathrm{h}$ ) and to $116.6 \pm 1.3 \mathrm{mEq} / 1$ in severe hyponatremia (DDAVP: $1 \mathrm{ng} / \mathrm{h}$ ), and were maintained from day 5 to the end of the experiment (Fig. 1). On day 13, plasma sodium levels in rats with moderate and severe hyponatremia were $124 \cdot 5 \pm 1 \cdot 0$ and $117 \cdot 4 \pm 0 \cdot 5 \mathrm{mEq} / \mathrm{l}$ respectively.

There was no obvious general symptom or abnormal behavior in either moderate or severe hyponatremia groups compared with the normonatremic group as assessed by the 38 tests using a modified Irwin's assessment test on day 5 (data not shown).

The walking time on the rotarod test were $35 \cdot 1 \pm 5 \cdot 4 \mathrm{~s}$ (normonatremia), $41 \cdot 3 \pm 5 \cdot 4 \mathrm{~s}$ (moderate hyponatremia), and $31.4 \pm 3.5 \mathrm{~s}$ (severe hyponatremia) respectively, and there was no statistical significant difference among the groups.

However, the step-through latency of hyponatremic rats was significantly reduced compared with that of the normonatremic rats. Although all rats in the normonatremic group stayed in the light compartment for more than $180 \mathrm{~s}$, almost all rats in moderate and severe hyponatremic groups moved into the dark compartment within $180 \mathrm{~s}$ (Fig. 2). The median value of step-through latency in the normonatremia group was $>180 \mathrm{~s}$, and reduced to $35.1 \mathrm{~s}$ in the moderate hyponatremia group and to $26 \cdot 3 \mathrm{~s}$ in the severe hyponatremia group. The reductions were statistically significant (Fig. 2).

Results in Study 1 showed that only impaired memory was observed in our hyponatremic model, although there was no general symptom, abnormal behavior, or impaired motor activity.

\section{Study 2: Effect of tolvaptan treatment on reduced step-through} latency of hyponatremic rats in PAV

Using the severe hyponatremia model, the effects of tolvaptan were evaluated. Beginning on day 4 , tolvaptan gradually and significantly elevated plasma sodium concentrations along with increasing tolvaptan doses $(0 \cdot 25,0 \cdot 5,1,2,4$, and $8 \mathrm{mg} / \mathrm{kg}$; Fig. 3). On day 10, tolvaptan increased the plasma sodium concentration to $133 \cdot 5 \pm 1 \cdot 0$ vs $115 \cdot 0 \pm 0.9 \mathrm{mEq} / 1$ in the vehicle-treated group.

Similar to results in Study 1, hyponatremia significantly reduced the step-through latency (median: $11 \cdot 0 \mathrm{~s}$ ) compared with the normonatremic group (median: $>180 \mathrm{~s}$; not all animals stayed in the light compartment in the normonatremia group in this study compared with animals in Study 1 (Fig. 2), likely due to individual behavior differences). Tolvaptan treatment prolonged the step-through latency in the hyponatremic group (median: 99.3 s; Fig. 4).

Plasma osmolality, hematocrit, and plasma electrolyte concentrations were determined after PAV on day 14 in this study as shown in Table 1. Plasma osmolality, hematocrit, and electrolyte concentrations were significantly lowered in hyponatremic, and were improved by tolvaptan. These data are consistent with the ability of tolvaptan to correct hyponatremia.

To identify potential mechanisms of hyponatremiamediated memory impairment, brain water content was determined. As shown in Fig. 5, hyponatremia significantly increased brain water content, indicating brain edema. Tolvaptan was able to partially normalize brain water content.

To further investigate the role of plasma sodium on memory, it was plotted against step-through latency for each animal in all the groups (Fig. 6). Rats in Study 1 and 2 were

Table 1 Effect of tolvaptan on plasma parameters in chronic hyponatremia after passive avoidance test on day 14 . The differences between the normonatremia and the hyponatremia vehicle, and between the hyponatremia vehicle and hyponatremia tolvaptan groups were analyzed by two-tailed $t$-test

\begin{tabular}{|c|c|c|c|c|c|}
\hline & $\begin{array}{l}\text { Osmolality } \\
\text { (mOsm/kg) }\end{array}$ & Sodium (mEq/l) & Potassium (mEq/l) & Chloride (mEq/l) & Hematocrit $(\%)$ \\
\hline \multicolumn{6}{|l|}{ Group } \\
\hline Hyponatremia vehicle & $242 \cdot 0 \pm 2 \cdot 8^{*}$ & $117 \cdot 0 \pm 1 \cdot 1^{*}$ & $4 \cdot 57 \pm 0 \cdot 08^{*}$ & $81 \cdot 9 \pm 1 \cdot 1^{*}$ & $37 \cdot 6 \pm 0 \cdot 7^{*}$ \\
\hline Hyponatremia tolvaptan & $274 \cdot 1 \pm 3 \cdot 0^{\dagger}$ & $132 \cdot 6 \pm 1 \cdot 4^{+}$ & $5 \cdot 14 \pm 0 \cdot 19^{\mp}$ & $97 \cdot 7 \pm 1 \cdot 4^{+}$ & $41 \cdot 3 \pm 0 \cdot 5^{+}$ \\
\hline
\end{tabular}

${ }^{*} P<0 \cdot 01$ compared with normonatremia group. ${ }^{\ddagger} P<0 \cdot 05,{ }^{+} P<0 \cdot 01$ compared with hyponatremia vehicle group. 


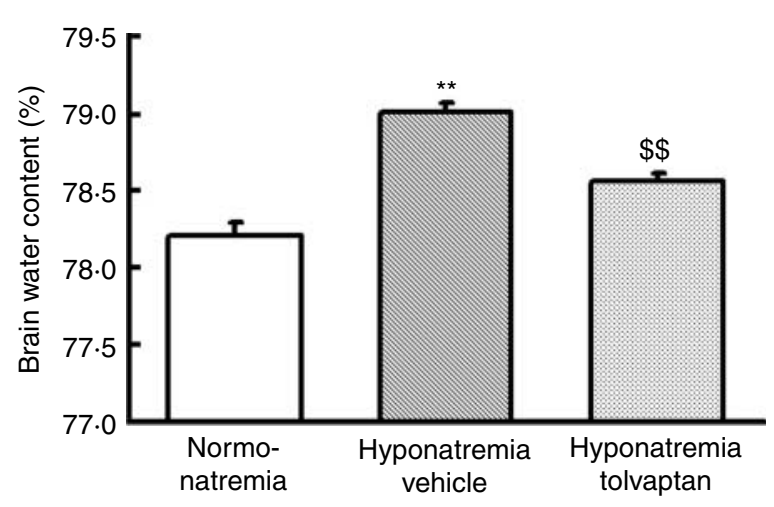

Figure 5 Effects of tolvaptan on brain water content. Values are expressed as the mean \pm S.E.M. of ten animals. The differences between the normonatremia and hyponatremia, and between hyponatremia vehicle and hyponatremia tolvaptan groups were analyzed by a two-tailed $t$-test. ${ }^{* *} P<0 \cdot 01$ compared with normonatremia group. ${ }^{\$} P<0 \cdot 01$ compared with hyponatremia vehicle group.

categorized in three groups ( $>135 \quad(n=24), 120-135$ $(n=20)$, and $<120 \mathrm{mEq} / 1(n=16))$ by their plasma sodium concentrations on the days when PAV was conducted. As shown in Fig. 6, step-through latency was significantly shortened depending on the degree of hyponatremia, and $135 \mathrm{mEq} / 1$ seems to be a critical level that can cause significant memory impairment.

These results showed that reduced step-through latency in hyponatremic rats was improved by the treatment of tolvaptan, a vasopressin $V_{2}$ receptor antagonist, and the improvement might be associated with normalization of plasma sodium concentrations.

Study 3: Effect of tolvaptan itself on step-through latency of normal rats in $P A V$

To determine whether tolvaptan itself directly influences memory, step-through latency of normonatremic rats in PAV with/without tolvaptan treatment was measured. In Study 3, foot shock condition $(0 \cdot 8 \mathrm{~mA}$ for $2 \mathrm{~s})$ of PAV was weaker than those in Study 1 and 2 ( $2 \mathrm{~mA}$ for $3 \mathrm{~s})$. In this condition, all rats moved into the dark compartment within $180 \mathrm{~s}$; and extension (or reduction) of time that rats stayed in the light compartment should be observed if the memory was influenced by tolvaptan itself. However, there was no difference in step-through latency between vehicle$(63 \cdot 6 \pm 25 \cdot 4 \mathrm{~s})$ and tolvaptan- $(57 \cdot 8 \pm 22 \cdot 5 \mathrm{~s})$ treated groups, suggesting that treatment with tolvaptan $(10 \mathrm{mg} / \mathrm{kg})$ itself did not influence to the memory of normal rats in PAV.

\section{Discussion}

At first, we tried to clarify whether 'asymptomatic' hyponatremia was really asymptomatic using a standard hyponatremic model in rats. There was no obvious general symptom or abnormal behavior (Irwin's test), and impairment of motor activity was not observed (rotarod test) in hyponatremic rats. From these results, our hyponatremia model seemed to be 'asymptomatic' even in the severe case. However, the step-through latency of hyponatremic rats was significantly reduced compared with the normonatremic rats in the PAV. Thus, an impairment of memory (or learning) was observed in rats with hyponatremia even in the moderate case.

Next, we performed PAV after plasma sodium correction by specific vasopressin $\mathrm{V}_{2}$ receptor antagonist tolvaptan, to examine whether impaired memory was caused by hyponatremia. When hyponatremia was partially normalized with tolvaptan, it was associated with an improvement of reduced step-through latency. In our data, vasopressin $\mathrm{V}_{2}$ receptor stimulation by DDAVP without water overload (not hyponatremic) did not impair PAV performance (data not shown). Furthermore, tolvaptan did not cross the blood-brain barrier; and tolvaptan itself did not influence the step-through latency as shown in Study 3. These data suggest that the improvement of step-through latency by tolvaptan treatment is not direct effect of vasopressin receptor antagonism but likely due to the normalization of plasma sodium.

In addition, our results indicate that a plasma sodium level of $135 \mathrm{mEq} / 1$ is critical. Below this level, impairment of memory occurs, and impairment of memory is observed in almost all rats when plasma sodium falls below $120 \mathrm{mEq} / \mathrm{l}$. Although further studies will be required to clarify which process of memory, such as memory acquisition, consolidation, and/or retrieval, are impaired by chronic hyponatremia,

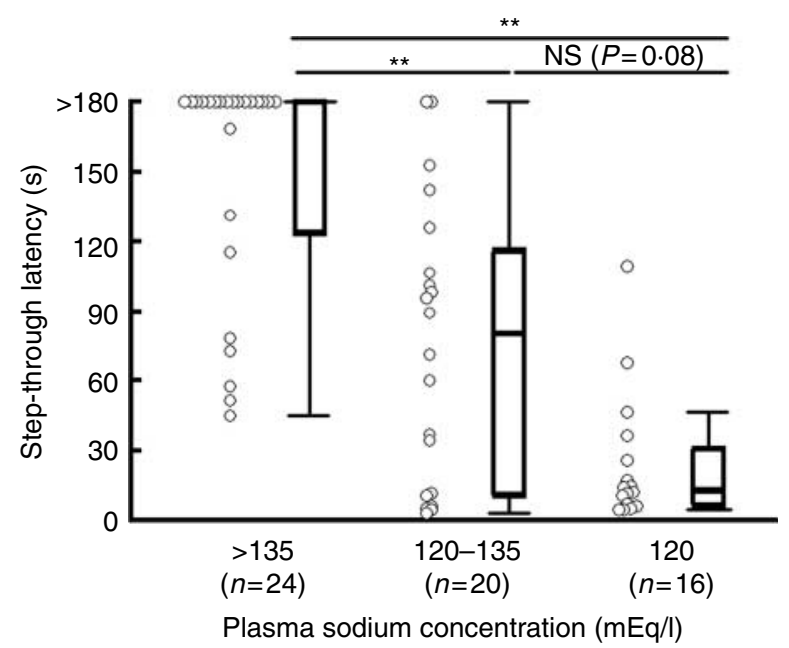

Figure 6 Relationship between step-through latency and plasma sodium concentration in hyponatremic rats. The individual values of 60 animals (rats in Study 1 and 2) were categorized in three groups ( $>135,120-135$, and $<120 \mathrm{mEq}$ groups) by their plasma sodium concentrations on the days when PAV was performed. The differences between the groups were analyzed by Steel-Dwass's test. ${ }^{* *} P<0 \cdot 01$ compared with $>135 \mathrm{mEq}$ group. The individual step-through latencies of animals (circle) and median with interquartile range (box and whisker) are shown. 
our data suggest that the state of hyponatremia may have an influence for memory processes without affecting general symptoms, behavior, or motor activity.

The mechanisms of this phenomenon are still to be determined, but the abnormalities of brain electrolytes and/or organic osmolytes and neurotransmitters may play important roles. It has been reported that several neurotransmitters strongly influence to PAV, e.g. dopamine (Doyle \& Regan 1993), glutamate (Danysz et al. 1988, Gandolfi et al. 1990, Venable \& Kelly 1990, Riekkinen et al. 1996), and acetylcholine (Piercey et al. 1987, Hiramatsu et al. 1998, Anglade et al. 1999).

In the chronic hyponatremia, brain cells export electrolytes and organic osmolytes including neurotransmitters to prevent excess swelling, resulting in decreased electrolytes and/or organic osmolytes and neurotransmitter (e.g. glutamine/ glutamate, GABA, aspartic acid, and glycine) content (Thurston \& Hauhart 1987, Lien et al. 1991, Verbalis \& Gullans 1991, Bedford \& Leader 1993, Massieu et al. 2004). The decrease of the neurotransmitters especially glutamine/ glutamate might strongly influence the memory. Furthermore, it has been reported that hypoosmolality inhibits glutamate and GABA uptake in rat brain synaptosome in vitro (Waseem et al. 2004).

In the present study, brain swelling (increased water content) and hypoosmolality was still observed on the days when PAV was performed in hyponatremic rats. Both brain cell swelling (which might change the physical environment around the neurons) and abnormalities in organic osmolytes (especially neurotransmitters) may have led to an impaired memory.

The other possible mechanism is the participation of angiotensin II (Ang II). In the SIADH model rats, it has been reported that plasma renin activity is lowered due to the mild volume expansion caused by the water retention (Song et al. 2004). There are some reports concerning the role of Ang II in memory and learning. Several reports have supported that Ang II impaired memory and learning, but conflicting results have also been reported, and the critical role of Ang II is not yet clear (reviewed by Gard (2002)). Anyway, if circulating Ang II level really plays important role in PAV, decreased Ang II level caused by hyponatremia may impair memory function, although effect of Ang II is still controversial.

In conclusion, this is the first study to demonstrate the association between chronic hyponatremia and memory deficit. These data also suggest that treatment of hyponatremia such as using $\mathrm{V}_{2}$ antagonists may be beneficial.

\section{Declaration of interest}

The authors are employees of Otsuka Pharmaceutical Co., Ltd which develops tolvaptan.

\section{Funding}

This research did not receive any specific grant from any funding agency in the public, commercial, or not-for-profit sector.

\section{Acknowledgements}

The authors express their sincere thanks to Dr Y Liu for his critical reading of this manuscript. The authors also thank Dr E Hosoki, Dr K Aihara, Dr T Futamura, Mr Y Toba, Mr T Ibuki, and Ms S Nakajima for their contribution and their excellent technical assistance.

\section{References}

Anglade F, Chapouthier G \& Galey D 1999 Intraseptal injection of scopolamine increases the effect of systemic diazepam on passive avoidance learning and emotionality in rats. Life Sciences 64 1553-1561.

Bedford JJ \& Leader JP 1993 Response of tissues of the rat to anisosmolality in vivo. American Journal of Physiology 264 R1164-R1179.

Danysz W, Wroblewski JT \& Costa E 1988 Learning impairment in rats by N-methyl-D-asparate receptor antagonists. Neuropharmacology 27 653-656.

Darbra S, Balada F, Martí-Carbonell MA \& Garau A 2004 Perinatal hypothyroidism effects on step-through passive avoidance task in rats. Physiology and Behavior 82 497-501.

Doyle E \& Regan CM 1993 Cholinergic and dopaminergic agents which inhibit a passive avoidance response attenuate the paradigm-specific increases in NCAM sialylation state. Journal of Neural Transmission 92 33-49.

Food and Drug Administration. FDA Labeling Information 2009 FDA web site [online], http://www.fda.gov/Drugs/default.htm.

Gandolfi O, Dall'Olio R, Roncada P \& Montanaro N 1990 NMDA antagonists interact with 5-HT-stimulated phosphatidylinositol metabolism and impair passive avoidance retention in the rat. Neuroscience Letters 113 304-308.

Gard PR 2002 The role of angiotensin II in cognition and behaviour. European Journal of Pharmacology 438 1-14.

Hiramatsu M, Murasawa H, Nabeshima T \& Kameyama T 1998 Effects of $\mathrm{U}-50,488 \mathrm{H}$ on scopolamine-, mecamylamine- and dizocilpine-induced learning and memory impairment in rats. Journal of Pharmacology and Experimental Therapeutics 284 858-867.

Hirano T, Yamamura Y, Nakamura S, Onogawa T \& Mori T 2000 Effects of the $\mathrm{V}_{2}$-receptor antagonist $\mathrm{OPC}-41061$ and the loop diuretic furosemide alone and in combination in rats. Journal of Pharmacology and Experimental Therapeutics 292 288-294.

Irwin S 1968 Comprehensive observational assessment: Ia. A systemic, quantitative procedure for assessing the behavioral and physiologic state of the mouse. Psychopharmacologia 13 222-257.

Lien YH, Shapiro JI \& Chan L 1991 Study of brain electrolytes and organic osmolytes during correction of chronic hyponatremia. Journal of Clinical Investigation 88 303-309.

Massieu L, Montiel T, Robles G \& Quesada O 2004 Brain amino acids during hyponatremia in vivo: clinical observations and experimental studies. Neurochemical Research 29 73-81.

Miller M 2006 Hyponatremia and arginine vasopressin dysregulation: mechanisms, clinical consequences, and management. Journal of the American Geriatrics Society 54 345-353.

Miyazaki T, Yamamura Y, Onogawa T, Nakamura S, Kinoshita S, Nakayama S, Fujiki H \& Mori T 2005 Therapeutic effects of tolvaptan, a potent, selective nonpeptide vasopressin $\mathrm{V}_{2}$ receptor antagonist, in rats with acute and chronic severe hyponatremia. Endocrinology 146 3037-3043.

Piercey MF, Vogelsang GD, Franklin SR \& Tang AH 1987 Reversal of scopolamine-induced amnesia and alterations in energy metabolism by the nootropic piracetam: implications regarding identification of brain structures involved in consolidation of memory traces. Brain Research 272 1-9.

Renneboog B, Musch W, Vandemergel X, Manto MU \& Decaux G 2006 Mild chronic hyponatremia is associated with fall, unsteadiness, and attention deficits. American Journal of Medicine 119 71.e1-71.e8.

Riekkinen M, Stefanski R, Kuitunen J \& Riekkinen P Jr 1996 Effects of combined block of alpha 1-adrenoceptors and NMDA receptors on spatial and passive avoidance behavior in rats. European Journal of Pharmacology 300 9-16. 
Sandhu HS, Gilles E, DeVita MV \& Michelis MF 2009 Hyponatremia associated with large-bone fracture in elderly patients. International Urology and Nephrology $\mathbf{4 1}$ 733-737.

Schrier RW, Gross P, Gheorghiade M, Berl T, Verbalis JG, Czerwiec FS, Orlandi C \& for the SALT Investigators 2006 Tolvaptan, a selective oral vasopressin $\mathrm{V}_{2}$-receptor antagonist, for hyponatremia. New England Journal of Medicine 355 2099-2112.

Song J, Hu X, Khan O, Tian Y, Verbalis JG \& Ecelbarger CA 2004 Increased blood pressure, ardosterone activity, and regional differences in renal $\mathrm{ENaC}$ protein during vasopressin escape. American Journal of Physiology $\mathbf{2 8 7}$ F1076-F1083.

Soupart A \& Decaux G 1996 Therapeutic recommendations for management of severe hyponatremia: current concepts on pathogenesis and prevention of neurologic complications. Clinical Nephrology 46 149-169.

Thurston JH \& Hauhart RE 1987 Brain amino acids decrease in choronic hyponatremia and rapid correction causes brain dehydration: possible clinical significance. Life Sciences 40 2539-2542.

Venable N \& Kelly PH 1990 Effects of NMDA receptor antagonists on passive avoidance learning and retrieval in rats and mice. Psychopharmacology 100 215-221.

Verbalis JG \& Gullans SR 1991 Hyponatremia causes large sustained reductions in brain content of multiple organic osmolytes in rats. Brain Research 567 274-282.
Verbalis JG, Zeltser D, Smith N, Barve A \& Andoh M 2008 Assessment of the efficacy and safety of intravenous conivaptan in patients with euvolaemic hyponatraemia: subgroup analysis of a randomized, controlled study. Clinical Endocrinology 69 159-168.

Waseem TV, Konev SV \& Fedorovich SV 2004 Influence of hypotonic shock on glutamate and GABA uptake in rat brain synaptosomes. Neurochemical Research 29 1653-1658.

Yamamura Y, Nakamura S, Itoh S, Hirano T, Onogawa T, Yamashita T, Yamada Y, Tsujimae K, Aoyama M, Kotosai K et al. 1998 OPC-41061, a highly potent human vasopressin $\mathrm{V}_{2}$-receptor antagonist: pharmacological profile and aquaretic effect by single and multiple oral dosing in rats. Journal of Pharmacology and Experimental Therapeutics 287 860-867.

Yu HY, Liu MG, Liu DN, Shang GW, Wang Y, Qi C, Zhang KP, Song ZJ \& Chen J 2007 Antinociceptive effects of paeoniflorin on bee venom-induced various 'phenotypes' of nociception and hypersensitivity. Pharmacology, Biochemistry, and Behavior 88 131-140.

Received in final form 1 April 2010
Accepted 14 April 2010

Made available online as an Accepted Preprint 14 April 2010 